\title{
DESARROLLO DE ALGORITMOS PARA EL MODELADO DE ESCULTURAS CON ARUNDO DONAX
}

\author{
DEVELOPMENT OF ALGORITHMS FOR MODELLING \\ SCULPTURES WITH ARUNDO DONAX
}

\author{
${ }^{1}$ Rene Nelson Alanoca Tarqui
}

\begin{abstract}
RESUMEN
El presente trabajo de investigación tiene como objetivo el desarrollo y la implementación de algoritmos 3D, mediante plugins de apoyo que actúan dentro del software $\mathrm{CAD}$; demostrando con ello, que se puede modelar de manera eficiente una escultura y hacer su comercialización más eficaz. Así mismo, tener información más detallada para la normalización de esculturas de Arundo Donax a través de hojas de cálculo. Los plugins son alimentados con datos recopilados de los cañaverales de Tacna, estos insumos ayudan a generar componentes que a su vez están formados por objetos $3 \mathrm{D}$ que derivan de primitivas del software CAD. El artista debe valerse de dichos componentes y modelar la escultura con el mejor ángulo, vía transformaciones necesarias en el entorno gráfico del software base, que para nuestro caso es 3D Studio Max 2012; el cual facilita una visión anticipada de la escultura en la computadora, con las medidas cuasi-reales. Como resultado de esta tesis, se puede construir y modelar de manera más eficiente las esculturas porque mediante los algoritmos 3D se reduce el tiempo de manera considerable en gabinete, aproximadamente el $880 \%$, mediante ahorro en cálculos, transformaciones y demás procesos que serían tediosos si dicha escultura se modelara de manera manual en el entorno del software base.
\end{abstract}

Palabras Clave: Algoritmos, Arundo, escultura, primitiva, software.

\begin{abstract}
The objective of this research is the development and implementation of $3 \mathrm{D}$ algorithms, through support plugins that act within the CAD software; demonstrating with it, that you can efficiently model a sculpture and make its marketing more efficient. In addition another objective was have more detailed information for the normalization of Arundo Donax sculptures through spreadsheets. The plugins are fed with data collected from the cane fields from Tacna, these inputs help to generate components that in turn are formed by $3 \mathrm{D}$ objects that derive from primitives of CAD software. The artist must use these components and model the sculpture with the best angle, via necessary transformations in the graphic environment of the base software, which for our case is 3D Studio Max 2012; which facilitates an anticipated view of the sculpture on the computer, with quasi-real measurements. As a result of this thesis, sculptures can be built and modeled more efficiently because 3D algorithms reduce time considerably in the cabinet, approximately $880 \%$, through savings in calculations, transformations and other processes that would be tedious if this sculpture will be modeled manually in the base software environment.
\end{abstract}

Keywords: Algorithms, Arundo, sculpture, primitive, software.

${ }^{1}$ Investigador del recurso caña hueca y sus aplicaciones. Ingeniero en Informática y Sistemas. Tacna- Perú. E-mail: renealanoca@gmail.com 


\section{INTRODUCCIÓN}

Tacna, por su situación geográfica, es rica en valles donde crece naturalmente el recurso denominado cafia hueca, cuyo nombre científico es Arundo Donax y que desde antaño la población ha sabido aprovecharlo para su modus vivendis (Egg, 1978).

En la actualidad se utiliza para elaborar esculturas sin pegamento y el artista las construye con todo lo que esto demanda: materia prima, tiempo, mano de obra, creatividad, etc. Tal es así que resulta una escultura apreciable; sin embargo, presenta ciertas debilidades ante la venta al publico cuando se requiere alguna modificación sugerida por parte del cliente; por tanto, los plugins evitan muchos contratiempos e impases, al elaborar una escultura digital por componentes que se puede modelar previamente antes de trabajarla de modo manual (Ramírez, 2003).

La tecnología está abordando varios aspectos del quehacer humano y el arte no es una excepción (Orozco, Chávez \& Chávez, 2006), por lo cual se ve favorecido con estos plugins que ayudarán al artista a elaborar una escultura más detallada para poder ofrecer y vender con certeza dicha pieza de arte a un potencial comprador. En estas tareas, el software CAD juega un papel importante.

Es crucial que se comprendan estas ideas y contribuyan al florecimiento de esa sinergia entre el estudiante y la tecnología (Moreno, 2003).

\section{MATERIALES Y MÉTODOS}

El método que se empleó para esta investigación fue el conocido como "Geometría Sólida Constructiva", que consta de la combinación de volúmenes que se traslapan al utilizar operaciones booleanas para la formación de un nuevo volumen (Hearn \& Backer, 2004).

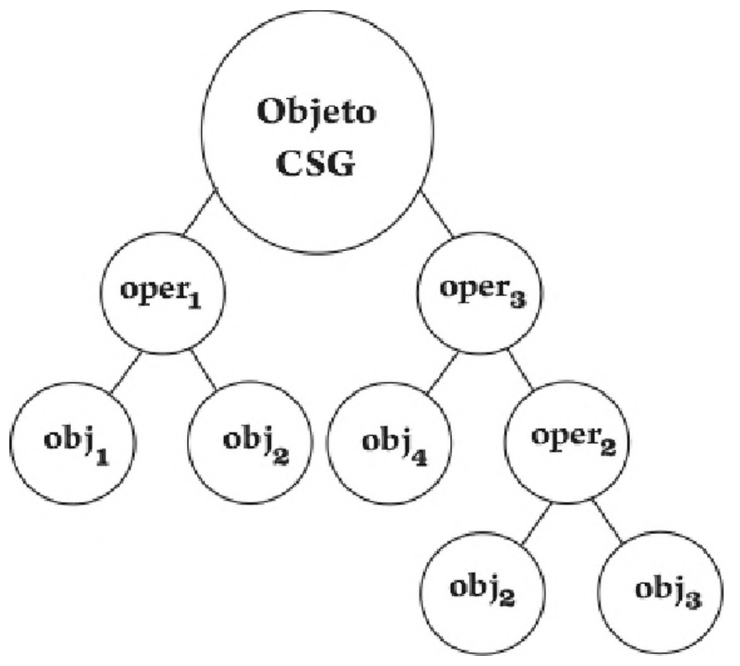

Figura 1. Árbol CSG para un objeto.

Fuente: Elaboración propia.

Esto se ve en la construcción de la escultura que tomamos como modelo representando a una "Avispa".

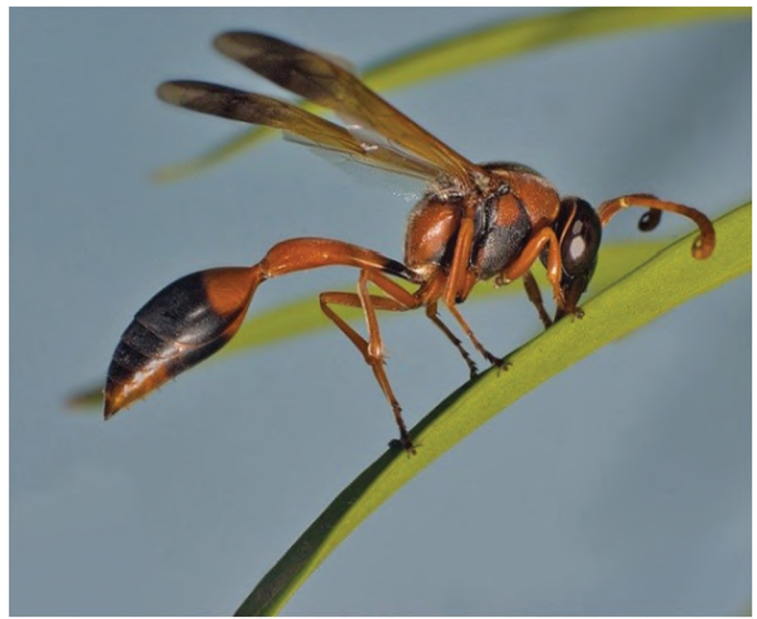

Figura 2. Insecto "Avispa".

Fuente: Elaboración propia.

\section{Análisis de datos}

Toma de datos de entrenudos (trozos tubulares) de Arundo Donax con diferentes diámetros entre el rango de $3 \mathrm{~mm}$ a $36 \mathrm{~mm}$

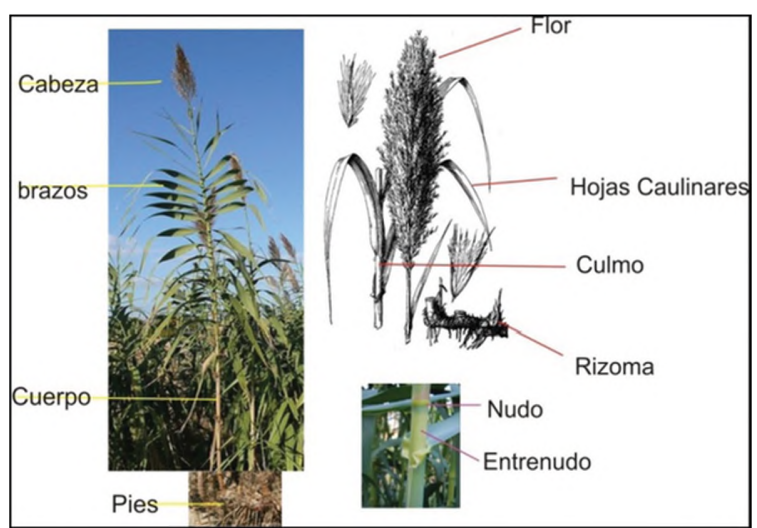

Figura 3. Arundo Donax (Caña Hueca) y sus partes. Fuente: Elaboración propia.

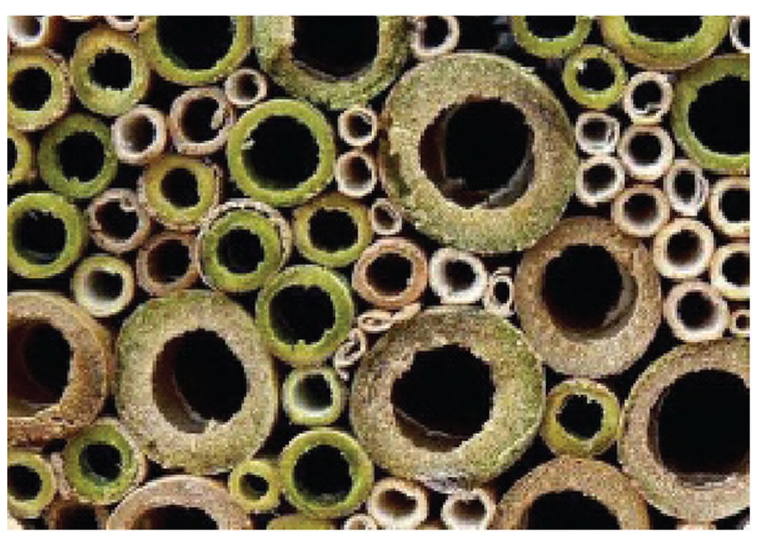

Figura 4. Diámetros de Arundo Donax. Fuente:Elaboración propia. 
Con la ayuda de un vernier se extrae las medidas de los diámetros exterior e interior, posteriormente se calcula la media aritmética entre la medida de la parte más corta y la parte más larga del corte transversal, ese resultado sería el diámetro exterior o interior buscado para los prototipos. También se extrae con una regla de metal graduada las medidas de la longitud de cada entrenudo (solo la parte útil) con el fin de poder calcular el máximo valor de longitud de cada pieza tubular utilizada en los prototipos.
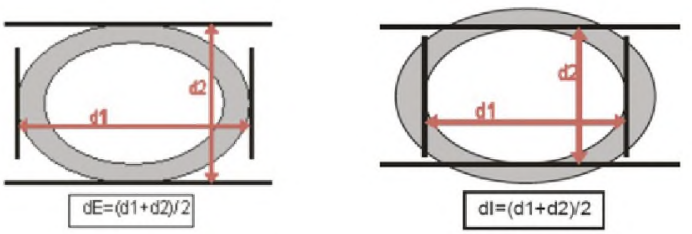

Figura 5. Medición con vernier de un entrenudo de Arundo Donax con sección no-circular.

Fuente: Elaboración propia.

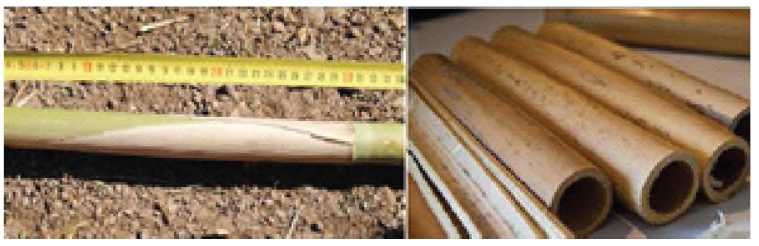

Figura 6. Medición de la longitud de trozos de caña (entrenudos).

Fuente: Elaboración propia.

E1 instrumento que se empleó para obtener la información fue una tabla de datos tomados de una muestra de 60 entrenudos de Arundo Donax y procesamiento de datos en Excel.

Tabla 1. Medición de la longitud de trozos de caña.

\begin{tabular}{|c|c|c|c|c|c|c|c|}
\hline \multirow[b]{2}{*}{$\begin{array}{l}\text { Fitrenudo } \\
\end{array}$} & \multicolumn{3}{|c|}{ Diámetro Exterior (mm) } & \multicolumn{3}{|c|}{ Diámetro Interior (mm,) } & \multirow[b]{2}{*}{ LONGTUD } \\
\hline & $\overline{\mathrm{DF} 1}$ & DE2 & $\begin{array}{c}\mathrm{DE} \\
\text { (Promedio) }\end{array}$ & Di1 & DI2 & $\begin{array}{c}\mathrm{DI} \\
\text { (Promedio) }\end{array}$ & \\
\hline 1 & 25,82 & 25,4 & 25,61 & 18,2 & 17,8 & 18 & 145 \\
\hline 2 & 25 & 24,7 & 24,85 & 18,1 & 7 & 17,55 & 147 \\
\hline 3 & 23,5 & 23,3 & 29,4 & 18 & 17,2 & 17,6 & 143 \\
\hline 4 & 21,8 & 21,5 & 21,65 & 16,7 & 14,9 & 15,8 & 132 \\
\hline 5 & 22,7 & 22,6 & 22,65 & 15,7 & 15,5 & 15,6 & 310 \\
\hline 6 & 21,6 & 21,4 & 21,5 & $\begin{array}{l}14,9 \\
14,9\end{array}$ & 15,6 & 15,25 & 296 \\
\hline 7 & 20,9 & 20,5 & 20,7 & 15,5 & 15 & 15,25 & 266 \\
\hline 8 & 19,2 & 18,9 & 19,05 & 13,6 & 13,3 & 13,45 & 223 \\
\hline 9 & 30,8 & 28.4 & 29,6 & 22,2 & 20,5 & 21,35 & 155 \\
\hline 10 & 7 & 6,9 & 6,95 & 4,1 & 4,1 & 4,1 & 50,5 \\
\hline 11 & 6,7 & 6,5 & 6,6 & 4,4 & 4 & 4,2 & 43,2 \\
\hline 12 & 4,9 & 4,8 & 4,85 & 3 & 2,5 & 2,75 & 25,5 \\
\hline 13 & 6,2 & 6 & 6,1 & 4 & 3,9 & 3,95 & 28,6 \\
\hline 14 & 5,3 & 5,1 & 5,2 & 3 & 2,9 & 2,95 & 60 \\
\hline 15 & 5,9 & 5,8 & 5,85 & 3,5 & 3,4 & 3,45 & 84 \\
\hline 16 & 7,5 & 7,3 & 7,4 & 4,4 & 4,4 & 4,4 & 67,6 \\
\hline 17 & 5,1 & 5 & 5,05 & 2,9 & 2,9 & 2,9 & 74,8 \\
\hline 18 & 3.4 & $\mathbf{3 . 4}$ & 3,4 & 1,4 & 1,39 & 1,395 & 28,5 \\
\hline 19 & 4,3 & 4,3 & 4,3 & 2,2 & 2,2 & 2,2 & 36,2 \\
\hline 20 & 15 & 14,6 & 14,8 & 11,7 & 11,6 & 11,65 & 90 \\
\hline
\end{tabular}

Fuente: Datos extraídos de entrenudos al azar.

La tabulación e interpretación de los datos se efectuó una vez recopiladas todas las lecturas y concluida la fase de investigación en campo. En cada unidad experimental se extrajo sus diámetros externos, diámetros internos y longitudes de los entrenudos que lo componen (De la Cruz, 2009); se halló una constante de relación entre las medidas de la estructura geométrica de los entrenudos (diámetro exterior, diámetro interior y longitud). Mediante esa constante de proporcionalidad se plantea conseguir una estimación cercana a la realidad de una pieza de escultura. Al ajustar la nube de puntos a una línea, utilizando el modelo de regresión lineal o mínimos cuadrados (Gutiérrez, 1981), se adapta mejor a los valores reales de cada pieza de escultura virtual. Existe cierto margen de error pues se detectaron diferencias algo significativas; esto se debe a variables que no son materia de estudio como el clima, suelo, agua, etc.

\begin{tabular}{c}
$\mathbf{y}=\mathbf{a}+\mathbf{b x}$ \\
$\mathrm{a}=-0,815024509$ \\
$\mathrm{~b}=0,751827162$ \\
\hline
\end{tabular}

$x$ : Diámetro exterior de trozo de Arundo Donax

$Y$ : Diámetro interior ajustado mediante regresión lineal

Figura 7. Ecuación de la recta de regresión Fuente: Elaboración propia

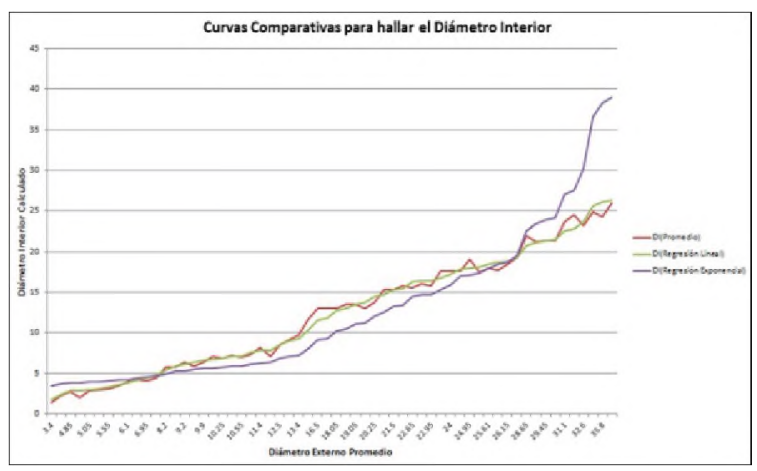

Figura 8. Curvas para estimar el diámetro interior. Fuente: Elaboración propia.

\section{Principales elementos tubulares}

Entre los principales elementos que nos proporciona la cañahueca tenemos:

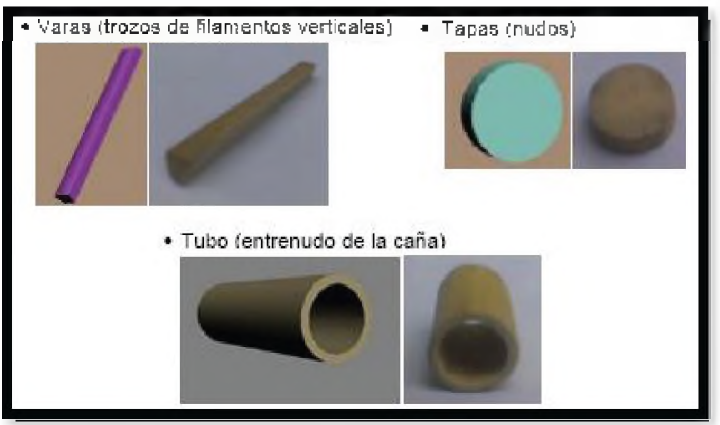

Figura 9. Elementos tubulares.

Fuente: Elaboración propia. 


\section{Identificación de primitivas}

Las primitivas son aquellas que comúnmente poseen los softwares de modelamiento y animación 3D y específicamente en el software usado en esta tesis: 3D Studio Max (García, 2006). Estas primitivas pueden encontrarse en una lista desplegable tanto en la sección del menú Create de la interfaz principal, como en la sección derecha de esta misma interfaz como una lista dinámica (Primitivas Estandar y Primitivas Extendidas). Las primitivas utilizadas para la generación de componentes de la escultura son: la primitiva "Tubo", "Cilindro", "Cilindro con chaflán"y "Caja".

\section{Identificación de los algoritmos}

Los algoritmos utilizados son la secuencia de pasos que se han de llevar a cabo en los procesos de construcción de componentes de la escultura. En su estructura, estos algoritmos trabajan con objetos 3D primitivos y realizan operaciones booleanas que el software base $3 D$ Studio ofrece; aquí se encuentra implícitamente el tema de proyecciones (Miranda, 1984). Por ejemplo, el algoritmo "Trompo" tiene como fin modelar un cuerpo sólido compuesto de varios segmentos de tubo con chaflán, concéntricos.

\author{
Algoritmo TROMPO (Recursivo) \\ 1. Inicio \\ 2. Leer Datos: DEXT (Diámetro Exterior) \\ LONG (Longitud de entrenudo) \\ POSINI (Posición de altura inicial respecto \\ a plano $\mathrm{XY}$ ) \\ 3. Si LONG $<=$ DEXT*7,146 \\ Entonces \\ Volver al paso 2 \\ /* Fin de Condición 3*/ \\ 4. Hacer BANDERA $\square 0$ \\ 5. SubRutina: TROMPO (DEXT, LONG, POSINI, \\ BANDERA) \\ 6. Obtener: Objeto 01 con medidas \\ Objeto 02 con medidas \\ Objeto $\mathrm{N}$ con medidas \\ Fin.
}

\section{Diagramas de flujo}

Para un desarrollo adecuado de los plugins, fue necesario hacer los diagramas de flujo correspondientes a cada módulo, ya que dichos diagramas facilitarán la construcción de la escultura objetivo.

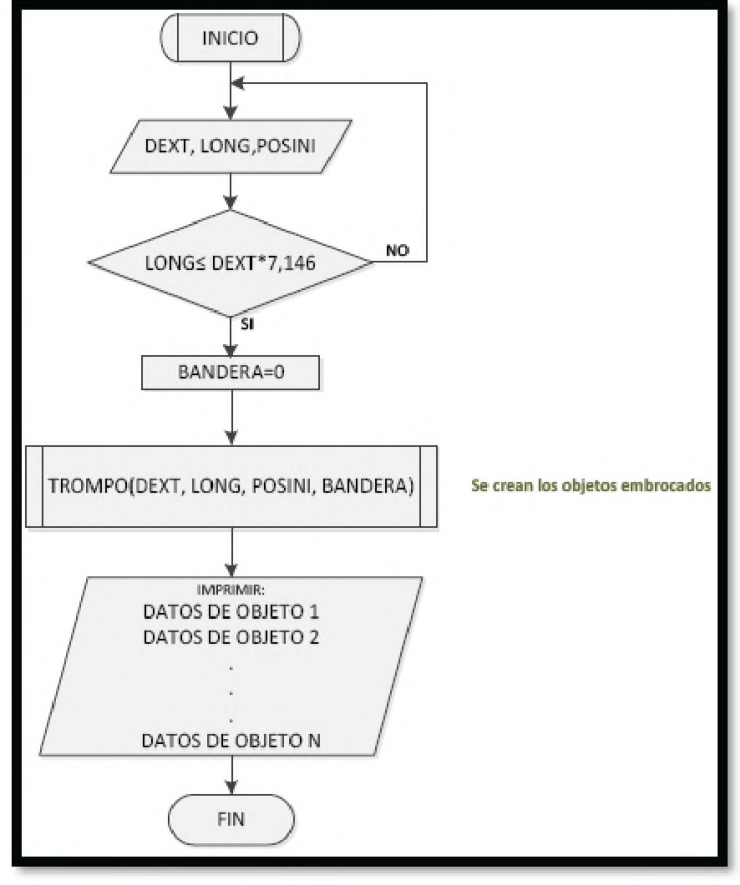

Figura 10. Diagrama de Flujo del algoritmo "Trompo".

Fuente: Elaboración propia.

\section{Implementación de componentes}

Plugin "Trompo"

Crea un cilindro grande y embroca un cilindro de menor diámetro externo que coincida con su diámetro interno y de mayor longitud, si se desea. Los datos de ingreso son el diámetro exterior del cilindro grande, el cual estará en un rango que se pueda trabajar en la técnica de la escultura de caña hueca (ej. $7 \mathrm{~mm}$ ). Además este cilindro grande no puede tener una longitud mayor a la existente tomada de la realidad (ej. $10 \mathrm{~mm}$ ) según su diámetro ingresado. Ubicamos la posición donde mostraremos el componente (ej. $X=10$ $\mathrm{mm}$ ), si se desea se le da un chaflán a cada borde de los tubos resultantes. Los cilindros generados, hasta el más pequeño, son producto de operación booleana de sustracción del mayor cilindro excepto el menor.

\section{Código Fuente de Plugin "Trompo"}

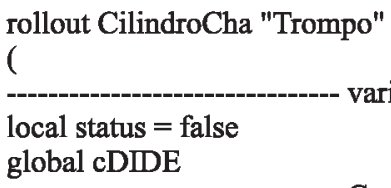

spinner diaext "Diametro Ext. range:[4,36,10] --[radio mín, radio máx, valor default] spinner posicionX "Pos X: " range:[5,30,10] --[filete mín, filete máx, valor default] spinner long "Longitud(mm): " range:[3,300,7] --[filete mín, filete max, valor default] group "Opciones de Chaflán" checkbox sele "Chaflán" pos:[40,90] 
radiobuttons rdo1 "'" pos:[40,120] width:23 height:32 enabled:false labels:\#("Ovalado", "Plano") default:1 columns: 1

label lab1 "Máx 10" pos:[135,106] enabled:false--[filete mín, filete máx, valor default

label lab2 "Mín 1" pos:[139,154] enabled:false slider chafle "Chaflan" pos:[175,80] range:[0.6,1.2,1.2] enabled:false width:40 height:24 orient:\#vertical

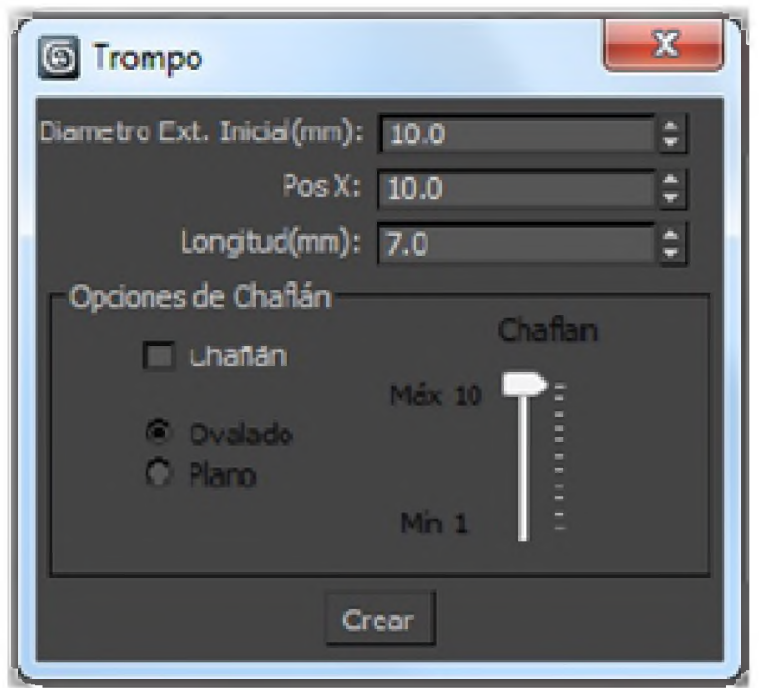

Figura 11. Formulario de ingreso de datos para generar un cuerpo sólido compuesto.

Fuente: Elaboración propia.
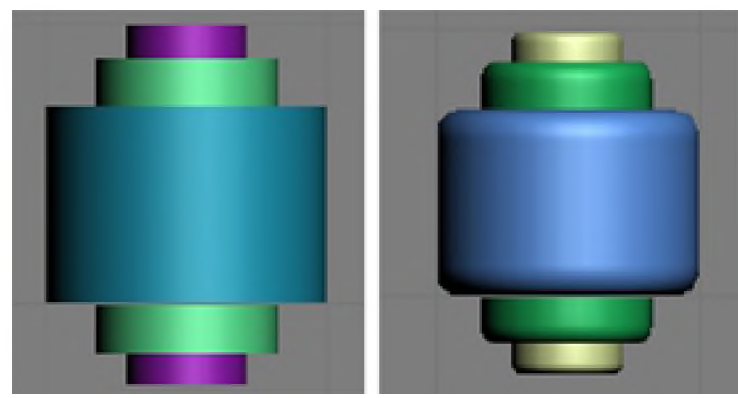

Figura 12. Tipos de componente generados por el plugin "Trompo". Fuente: Elaboración propia.
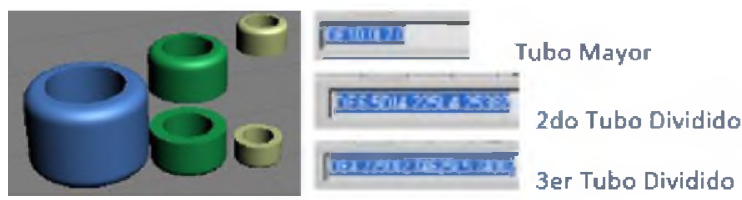

Figura 13. Objetos y sus datos internos generados por el plugin "Trompo".

Fuente:Elaboración propia.

\section{Construcción y modelamiento de prototipos virtuales}

Para modelar una escultura construida a través de cada componente generado por los plugins, pondremos de muestra la escultura de una avispa. Cada pieza será modelada manualmente en las vistas del software CAD a fin de ubicar sus coordenadas respectivas (Alva, F., s/f). Cuando el prototipo está listo, se abstrae las medidas de las partes componentes para ubicar -en tanto se mide con el vernier calibrado- dichas piezas en la naturaleza (en los culmos de la caña hueca) y trabajarlas para obtener la escultura final de caña a presión. La construcción de la escultura digital 
comienza desde el boceto inicial en papel (Zatonyi, 2002), luego se realizan trazos con lápiz para obtener direcciones de la caña sobre la orientación del dibujo. Posteriormente, con cierta agudeza, se debe separar en bloques la escultura final para ensamblarla por partes. Cada bloque obedece al criterio siguiente: No es recomendable ni posible armar toda la pieza de un solo paso, pues se presentarían turbulencias en los elementos ya ensamblados, en tanto se busca insertar nuevos componentes. En cada bloque algunos componentes SCG no son más que objetos resultantes de aplicar algoritmos 3D, quedando como resultado un solo objeto. Todo esto se muestra en un árbol donde las hojas terminan siendo objetos o componentes, mas no bloques.

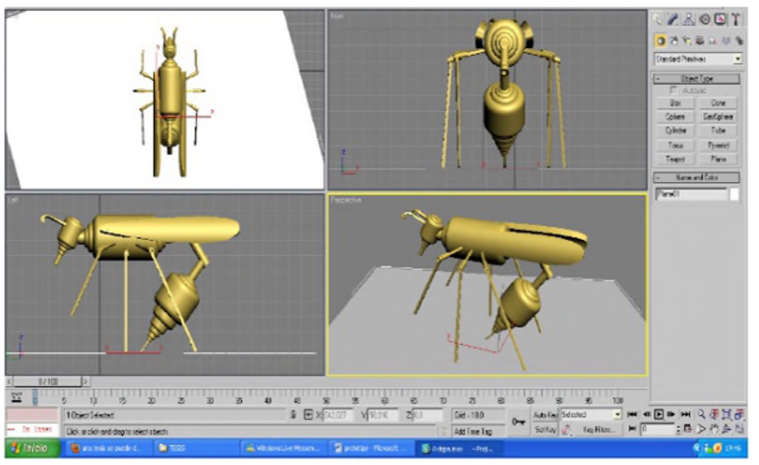

Figura 14. Modelamiento de la escultura digital en el entorno 3D Studio Max.

Fuente: Elaboración propia.

\section{RESULTADOS}

Los resultados logrados con el uso de estos algoritmos de utilidad para generar componentes que luego serán integrados a la escultura final, haciendo uso de transformaciones matriciales (Rotar/Mover), se pueden observar en el siguiente versus con un $95 \%$ de proximidad a la escultura final física. A continuación, se presenta un cuadro comparativo que demuestra por qué se deben utilizar estos algoritmos en el modelado de esculturas de arundo donax. Se debe tener en cuenta que para hacer el modelo con o sin algoritmos, es indispensable tener los datos estadísticos a disposición, y así estimar manual o automáticamente las medidas a ingresar para generar luego cada parte componente de la escultura deseada.

\section{Formula para obtener diámetro interior (Ec.} Recta Regresión Lineal)

\section{$D I=0,815024509+0,751827162 * D E$}

Donde: DI: Diámetro interno del entrenudo de caña. DE: Diámetro externo del entrenudo de caña.
Tabla 2. Modelado manual vs modelado con algoritmos $3 D$.

\begin{tabular}{|c|c|c|c|c|c|c|c|}
\hline \multirow[b]{2}{*}{ Componente } & \multicolumn{3}{|c|}{ Auromásico } & \multicolumn{3}{|c|}{ Manual } & \multirow[b]{2}{*}{$\begin{array}{c}\text { Diterencia } \\
\text { (min) }\end{array}$} \\
\hline & $\begin{array}{l}\text { Hora } \\
\text { Inicio }\end{array}$ & Mord Fin & $\begin{array}{c}\text { Tiempo } \\
(\min )\end{array}$ & $\begin{array}{l}\text { Hord } \\
\text { Inicio }\end{array}$ & Hord Fin & $\begin{array}{c}\text { Tlempo } \\
\text { (min) }\end{array}$ & \\
\hline Ala de Avispa & 10:38am & $10: 40 \mathrm{am}$ & 2 & 9:36am & 9:59am & 23 & 21 \\
\hline Pata de Avispa & $11: 36 \mathrm{am}$ & $11: 38 \mathrm{am}$ & 2 & $11: 08 \mathrm{am}$ & $11: 36 \mathrm{~mm}$ & 28 & 26 \\
\hline Abdomen de Avispa & $12: 22 \mathrm{pm}$ & $12: 27 \mathrm{pm}$ & 5 & $11: 41 \mathrm{am}$ & $12: 21 \mathrm{pm}$ & 40 & 35 \\
\hline Tapa de Cabeza Avispa & $12: 35 \mathrm{pm}$ & $12: 36 \mathrm{pm}$ & 1 & $12: 28 \mathrm{pm}$ & $12: 35 \mathrm{pm}$ & 7 & 6 \\
\hline TOTAL DEMORA & & & 10 & & & 98 & 88 \\
\hline TOTAL $\%$ & & & $100 \%$ & & & $980 \%$ & $880 \%$ \\
\hline
\end{tabular}

Fuente: Elaboración propia.

En la tabla anterior se hace una comparación entre los patrones identificados en la escultura "Avispa" generados por algoritmos y los que se llevaron a cabo de manera manual, incluyendo las transformaciones que estos demanden, así como las medidas de cada objeto. Estos patrones se representan como componentes u objetos CSG, y son resultado de aplicar los plugin que devienen de los algoritmos 3D empleados.

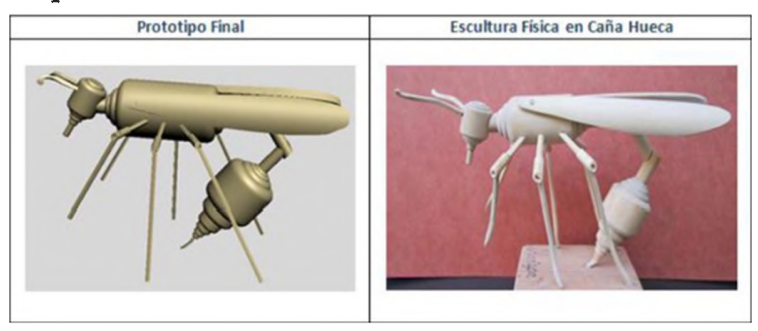

Figura 15. Prototipo final vs. escultura física en caña. Fuente: Elaboración propia.

Por otra parte, mediante una evaluación Costo/Beneficio, estos prototipos muestran una rentabilidad en ahorro para el escultor a la hora de ofrecer en venta cada escultura. Estos modelos digitales con las medidas precisas de Arundo Donax facilitan su envío a otros lugares del mundo sin necesidad de transportar las esculturas físicas (Alanoca, 2014).

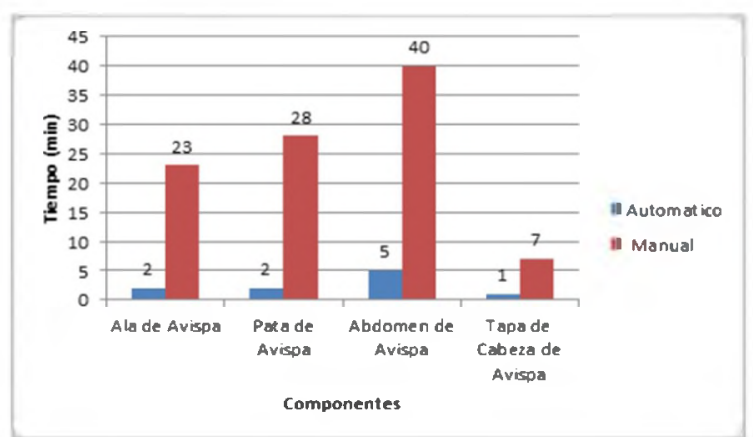

Figura 16. Cuadro comparativo de elaboración de componentes y Objetos CSG.

Fuente:Elaboración propia.

Se menciona además que con el plugin Extractor se captura toda la información de los objetos en el entorno gráfico de 3D Studio Max, como medidas, color, y coordenadas para ser mostrada en una hoja de cálculo. 
Tabla 3. Hoja de cálculo con la información importada desde 3DS Max de 05 objetos del plugin TROMPO.

\begin{tabular}{|c|c|c|c|}
\hline oneta & MOMGRE Y MEOHOLS & conom & Poardenadas \\
\hline 1 & 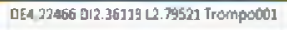 & $5 \% 65951$ & {$[1,20,10.4901]$} \\
\hline 2 & DE 5.70325 94. .72455 L1.71788 Trompo0001 & 11188598 & {$[1,20,16.7894)$} \\
\hline 3 & DEA 22466 [12.36]19L2.79521 Tremps & 11607185 & $\{1,20, T, 11293)$ \\
\hline 4 & DE5. 70325 g14.22465 L3.77788 Tromps & 1211745 & {$[1,20,0,35162]$} \\
\hline 5 & 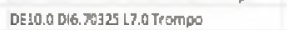 & 13958871 & {$[1,20,18]$} \\
\hline
\end{tabular}

Fuente: Elaboración propia.

\section{DISCUSIÓN}

Respecto de la hipótesis planteada en el presente trabajo de investigación, sobre si el uso de algoritmos 3D influye en el modelamiento de esculturas con Arundo Donax, se verifica su influencia positiva en el análisis del costo beneficio, así como en el ahorro de tiempo, gracias a la labor geométrica desarrollada en la elaboración de una escultura física mediante el uso de la computadora.

En el marco teórico de la investigación se ha definido todos los aspectos necesarios para facilitar la elaboración de una escultura de Arundo Donax, utilizando algoritmos 3D, como medio para mejorar el cumplimiento de los objetivos trazados.

\section{CONCLUSIONES}

Se analizaron correctamente los datos específicos de la estructura geométrica de Arundo Donax para su introducción en plugins.

Se implementó algoritmos 3D mediante plugins de utilidad empotrados en el entorno del software 3D Studio Max, que realicen cálculos y operaciones booleanas entre objetos 3D.

Se construyó de manera confiable y óptima un modelo de prototipo de la escultura de una avispa, con gran ahorro de tiempo.

\section{REFERENCIAS BIBLIOGRÁFICAS}

Alanoca, R. (2014). Desarrollo de algoritmos 3d para el modelado de esculturas con arundo donax. Tacna, Perú:UNJBG.

Alva, F. (S/F)Geometría Contemporánea. Lima, Perú: San Marcos. (Item: Construcción y modelamiento de prototipos virtuales)

De la Cruz, M. (2009). Estudio del comportamiento mecánico de ductos sometidos a diferentes condiciones de carga mediante modelado numérico. Escuela Superior de Ingeniería y Arquitectura. México, 25-31. (Item: Análisis de datos)

Egg, E. (1978). Introducción a las Técnicas de Investigación Social (7 ed.). Buenos Aires, Argentina: HVMANITAS. (Item: Introducción)

García, J. (2006). 3D Studio MAX 8 (1 ed.). Perú: Megabyte. (Item: Identificación de primitivas)

Gutiérrez, J. (1981). Estadística. España: CULTURAL.

(Item: Análisis de datos)

Hearn, D. y Backer, P. (1994). Gráficas por Computadora (2 ed.). México: Prentice Hall Inc.

Hearn, D. y Backer, P. (2004). Gráficos por Computadora con OpenGL (3 ed.). España: Prentice Hall. (Item: Materiales y Métodos)

Miranda, A. (1982). Geometría Descriptiva (4 ed.). Perú:UNI.(Item: Construcción y modelamiento de prototipos virtuales)

Miranda, A. (1984). Geometría Descriptiva (5 ed.). Perú:UNI.

Moreno, L. (2003). Instrumentos Matemáticos Computacionales. Cinvestav-IPN. México, 18, 1-128. (Item: Introducción)

Orozco, A. y Chavez, M. y Chavez, J. (2006). Informática Uno (1ra ed.). México: Thomson.

Ramírez M. (2003). La Ilusión en el Arte del Renacimiento a la Realidad Virtual (1 era ed; Addenda $\mathrm{N}^{\circ}$ 07). México: CENIDIAP. (Item: Introducción)

Zatonyi, M. (2002). ¿Realidad Virtual? Argentina: Ediciones Geka. 